\title{
Deflection Measurement for Bridges Based on Secant Inclination
}

\author{
Yi Wu $\mathbf{u}^{1}$, Jing $\mathbf{L} \mathbf{i}^{2}$ \\ ${ }^{1}$ Department of Civil Engineering, Guangdong Polytechnic of Water Resources and Electric Engineering, Guangzhou, China \\ ${ }^{2}$ School of Civil and Transportation Engineering, Guangdong University of Technology, Guangzhou, China \\ Email: 125372329@qq.com
}

How to cite this paper: $\mathrm{Wu}, \mathrm{Y}$. and $\mathrm{Li}, \mathrm{J}$. (2021) Deflection Measurement for Bridges Based on Secant Inclination. Open Journal of Civil Engineering, 11, 427-433. https://doi.org/10.4236/ojce.2021.114025

Received: August 24, 2021

Accepted: December 20, 2021

Published: December 23, 2021

Copyright $\odot 2021$ by author(s) and Scientific Research Publishing Inc. This work is licensed under the Creative Commons Attribution International License (CC BY 4.0).

http://creativecommons.org/licenses/by/4.0/

\begin{abstract}
Deflection is a significant indicator of bridge's strength and its whole stiffness, so the research on deflection measurement is an important aspect of bridge health monitoring. There have existed many measurement methods of bridge deflection so far, while inclination method is gradually catching more and more attention for its fair obviously comprehensive advantages. However, the inclination method at present focuses on measuring the rotation of bridge's section at testing point, that is, the tangent angle of deflection curve. With the tangent angle, the deflection curve can be determined by the methods of curve fitting or (and) integration or conjugate beam. The methods mentioned above, are not only complicated in calculation, but also bad in accuracy. The deflection measurement method proposed by this paper is based on measuring the inclination of two points initiatively in horizontal line, that is, the secant angle of the deflection curve, and on the simple triangle function operation. The proposed method is simple in theory, but good in accuracy for either static or dynamic load. The numerical simulation suggests that the error of the proposed method is less than $1 \%$.
\end{abstract}

\section{Keywords}

Bridge, Deflection, Inclination, Secant, Measurement

\section{Introduction}

Effective monitoring of the health of the bridge and taking appropriate maintenance measures when necessary are important measures to eliminate hidden dangers in bridge safety and prevent safety accidents on the bridge. Because the deflection is an important index reflecting the bearing capacity and stiffness of a bridge, therefore deflection measurement is an important work of bridge health 
monitoring. However, due to the factors of different bridge scale, environment, measurement duration, and requirements for the accuracy of measurement, not all deflection measurement methods are suitable for measuring the deflection of a specific bridge. Therefore, the deflection test has always been the key point and difficulty in the work of a bridge test. At present, scientific and technical personnel around the world have developed a variety of bridge deflection measurement methods, such as dial indicator method, pendant method, precision level measurement method, photoelectric imaging and CCD (Charge-Coupled Device) method, total station measurement method, connecting tube measurement method, GPS (Global Positioning System) method, laser imaging method, PSD (Position Sensitive Detector) laser measurement method, acceleration sensor method, conjugate beam method based on long gauge strain sensor, and displacement sensor direct measurement method [1] [2]. However, these methods have disadvantages of one kind or another, such as the need for reference points, low accuracy, expensive price, or unsuitable for dynamic or long-term monitoring. The inclination method has gradually received widespread attention due to its obvious comprehensive advantages, such as no need for a static reference point, high measurement efficiency, simultaneous measurement of dynamic and static deflection, low frequency characteristics, and good transient response capabilities. However, the current inclination method is mainly to measure the angle of the cross section of the measuring point when the bridge is deformed, that is, the tangent angle of the deflection curve, and then calculate the deflection of the bridge by curve fitting or (and) integration or conjugate beam methods [3] [4] [5] [6]. These methods are not only computationally complicated, but also have low accuracy. In this paper, the deflection of the measured point can be obtained by measuring the inclination angle of the connection between two points on the horizontal line of the bridge, that is, the secant angle of the deflection curve, and then using simple trigonometric function calculations. The proposed deflection measurement method is not only simple, but also highly accurate. Numerical simulation results show that the error of the proposed method is within $1 \%$.

\section{Principle of Secant Inclination Deflection Measurement}

The principle of measuring the bridge deflection by the secant inclination method is shown in Figure 1. The horizontal dashed line in the figure indicates the position of the bridge before deformation, and the arc-shaped solid line indicates the position after deformation, $i$ (in this case $i=0,1,2,3,4$ ) is the position of the measuring point, where $i_{0}$ and $i_{\mathrm{n}}$ (in this example $i_{0}=0, i_{\mathrm{n}}=4$ ) are also the position of the support, $f_{\mathrm{i}}$ is the deflection corresponding to the $i$-th measuring point, and $\Delta f_{\mathrm{i}}$ is the $i+1$-th measuring point Relative to the deflection increment of the $i$-th measuring point, $l$ is the span of the bridge, $n$ is the number of equal divisions of the measured points of the bridge, and $\Delta x$ is the horizontal distance between two adjacent measuring points before deformation, namely: 


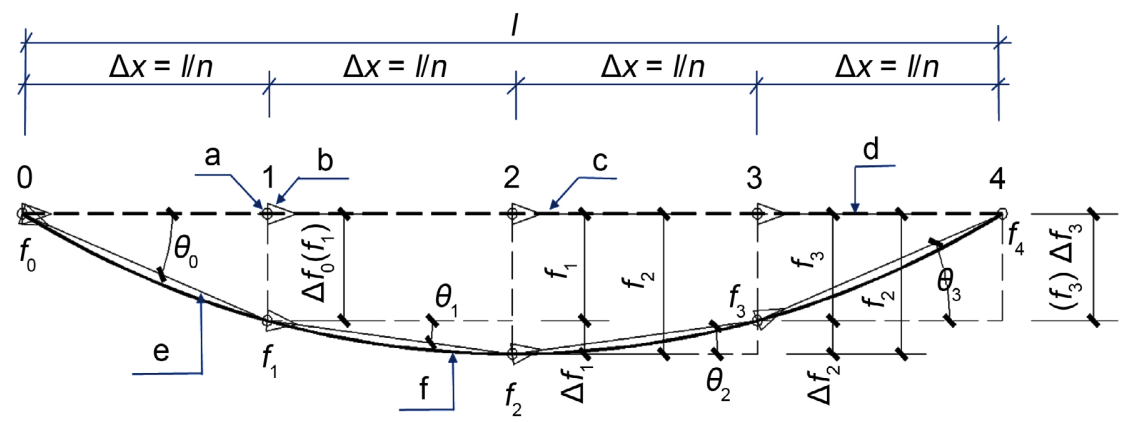

Notice:

1) $n=4$ in this paper;

2) Inclinometers installed on elastic chord are for measuring inclination of the chord;

3) Notation:

a-anchor point; b-inclinometer; c-beam position before deformation;

$\mathrm{d}$-chord position before deformation; e-chord position after deformation;

f-beam position after deformation.

Figure 1. Principle of deflection measurement by secant inclination method.

$$
\Delta x=l / n
$$

In actual measurement, a linear chord can be erected between adjacent measuring points (i.e. each internode). One end of the chord is restrained by a hinge support, and the other end is restrained by a roller support to ensure that the chord can be free to expand and contract as the bridge deformed, without bending deformation to affect the accuracy of angle measurement. The inclinometer is fixed on the chord. Before the bridge is deformed, or when the chord is in a horizontal state, the inclinometer's reading is 0 . After the bridge is deformed, the inclinometer's reading $\theta_{\mathrm{i}}$ is the inclination angle of the connecting line between the $i+1$ th and the $i$-th measuring points, namely the inclination angle of the secant line of the deflection curve.

It is easy to know from Figure 1 that the deflection increment of the measuring point of $i+1$ th relative to the $i$-th is:

$$
\Delta f_{i}=\Delta x \cdot \tan \theta_{i}
$$

The deflection corresponding to the $i$-th measuring point is:

$$
f_{i}=f_{i-1}+\Delta f_{i-1}
$$

The deflection $f_{0}$ of the initial measurement point at the left support can be directly measured by a displacement meter erected on the pier (the pier can be approximately regarded as a static reference point). After $f_{0}$ is measured, the measured inclination angle $\theta_{\mathrm{i}}$ of each chord, as well as formula (2) and formula (3) can be used to obtain the deflection of each measuring point.

It can be seen from Figure 1 that the cross-section of the bridge will rotate after the deformation, so that the measuring point is not right under the point where before the bridge deforms, resulting in an error in the deflection calculated according to the above formula. A test of this error will made below by using numerical simulation method. 


\section{Verification of the Measured Deflection Value Under Static Load}

In this section, a large-scale commercial finite element software, ANSYS, will be used to numerically simulate a simply supported steel beam under static load to verify the accuracy and reliability of the proposed secant inclination deflection measurement theory. The span of the simply supported beam is $l=10 \mathrm{~m}$, the width of the rectangular section is $b=0.2 \mathrm{~m}$, the section height is $h=0.5 \mathrm{~m}$, the steel's elastic modulus is $E=2 \times 1011 \mathrm{~N} / \mathrm{m}^{2}$, its Poisson's ratio is $\mu=0.3$, and its density is $\rho=7800 \mathrm{~kg} / \mathrm{m}^{3}$. The plane 42 plane stress element is used in simulation model, and the finite element model is shown in Figure 2.

Here, three load cases are studied, namely the deflection of the concentrated load acting on the $1 / 2$ span (that is, the middle span) and the $1 / 4$ span, and the uniform load. The deflections at the $1 / 2$ span and $1 / 4$ span are investigated. Set the concentrated load $P=10,000,000 \mathrm{~N}$ and the uniform load $q=1,000,000$ $\mathrm{N} / \mathrm{m}$. In the numerical simulation, the secant inclination is calculated with the coordinates between the two measuring points after the bridge is deformed. The analysis results are shown in Table 1 . It can be seen from Table 1 that the maximum error is only $0.91 \%$, which is less than $1 \%$. Because the actual deflection-span ratio limit $1 / 600$ [7] is much smaller than the ratio $1 / 16.7$ of this example, the actual error should be much smaller than the error of this example.

\section{Numerical Verification of Deflection Measurement under Dynamic Load}

It can be seen from the previous section that the largest error is the mid-span

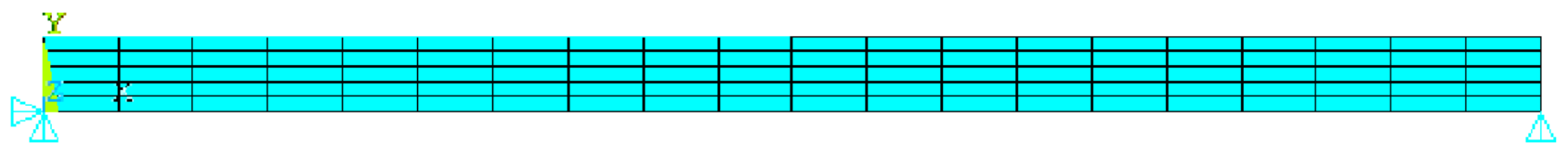

Figure 2. ANSYS finite element model.

Table 1. Numerical verification of deflection calculation under static load.

\begin{tabular}{|c|c|c|c|c|c|c|}
\hline & $\begin{array}{l}\text { Deflection of } \\
\text { mid-span } \\
\text { under } \\
\text { concentrated } \\
\text { load on } \\
\text { mid-span }\end{array}$ & $\begin{array}{l}\text { Deflection of } \\
\text { mid-span } \\
\text { under } \\
\text { concentrated } \\
\text { load on } \\
1 / 4 \text { span }\end{array}$ & $\begin{array}{l}\text { Deflection of } \\
1 / 4 \text { span under } \\
\text { concentrated } \\
\text { load on } 1 / 4 \\
\text { span }\end{array}$ & $\begin{array}{l}\text { Deflection of } \\
1 / 4 \text { span under } \\
\text { concentrated } \\
\text { load on } \\
\text { mid-span }\end{array}$ & $\begin{array}{l}\text { Deflection of } \\
\text { 1/4 span } \\
\text { under } \\
\text { uniform load }\end{array}$ & $\begin{array}{c}\text { Deflection } \\
\text { of mid-span } \\
\text { under } \\
\text { uniform load }\end{array}$ \\
\hline $\begin{array}{l}\text { Deflection from } \\
\text { proposed theory } \\
\text { (m): }\end{array}$ & -0.48089 & -0.37457 & -0.34598 & -0.38703 & -0.04939 & -0.61296 \\
\hline $\begin{array}{l}\text { Deflection from } \\
\text { ANSYS (m): }\end{array}$ & -0.47761 & -0.37190 & -0.34416 & -0.38555 & -0.04940 & -0.60745 \\
\hline Error & $0.69 \%$ & $0.72 \%$ & $0.53 \%$ & $0.39 \%$ & $-0.02 \%$ & $0.91 \%$ \\
\hline
\end{tabular}




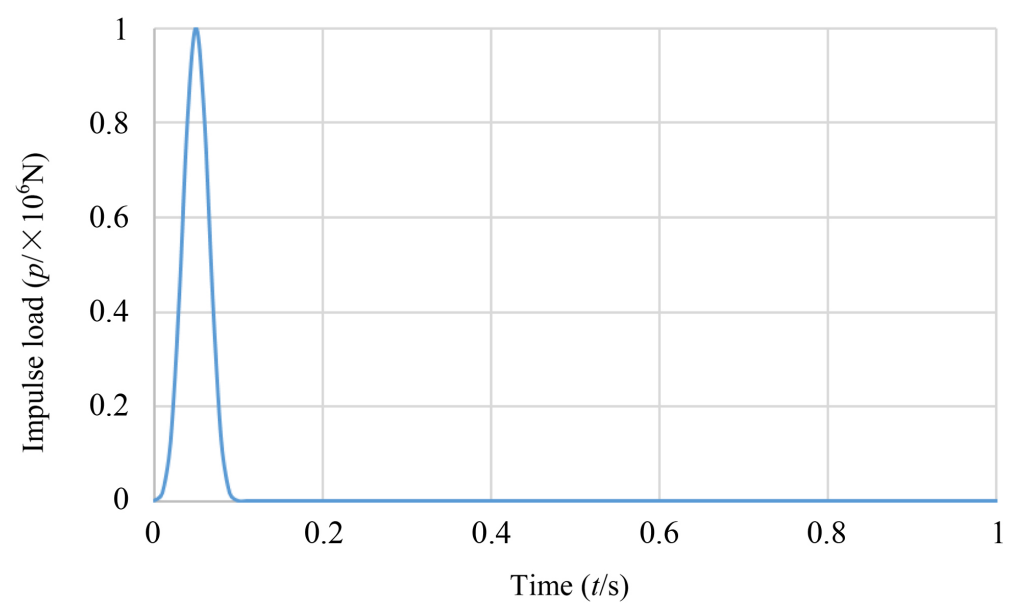

Figure 3. Pulse load curve.

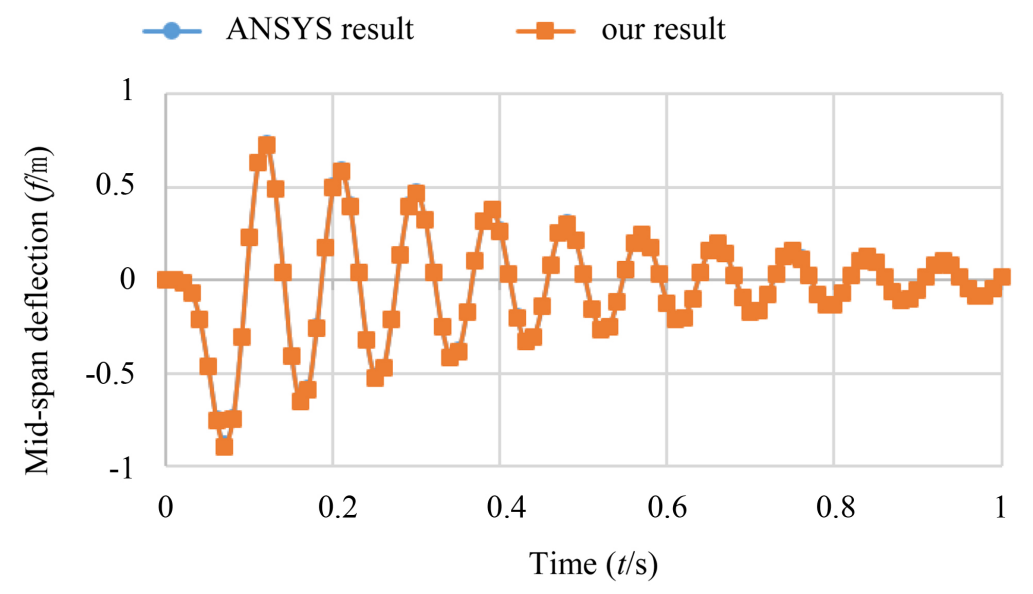

Figure 4. Verification of deflection calculation under impulse load.

deflection under uniform load, so this section will study the difference between the deflection calculation theory and numerical calculation results of the above simply supported beams under uniform impulse load. The pulse load used here takes the following form [8]:

$$
\begin{aligned}
p(t)= & 16 \times 10^{6} \times(G(\tau)-4 G(\tau-0.25)+6 G(\tau-0.5) \\
& -4 G(\tau-0.75)+G(\tau-1.0))
\end{aligned}
$$

where, $\tau=t / T_{0}, G(\tau)=\tau^{3} \cdot H(\tau), H(\tau)=\left\{\begin{array}{ll}0, & \tau<0 \\ 1, & \tau \geq 0\end{array}, \quad t, T_{0}\right.$ are calculation time and pulse duration respectively. The pulse load curve and the numerical verification results are shown in Figure 3 and Figure 4, respectively. It can be seen from Figure 4 that the deflection calculation scheme proposed in this paper also has good accuracy for dynamic loads.

\section{Conclusions}

In this paper, the bridge deflection is calculated by measuring the inclination an- 
gle of the line connecting adjacent measuring points on the horizontal line of the bridge, that is, the inclination angle of the secant line on the deflection curve. Not only is the method simple, easy to operate and realize, but also the numerical simulation results show that, whether under static load or dynamic load, it has high accuracy.

Numerical simulation results show that for the example model in this article, the error of the bridge deflection measurement method based on the secant inclination angle is within $1 \%$.

Since the deflection of the calculation example in this paper is as high as $1 / 16.7$ of the bridge span, while the deflection limit specified by the bridge code is only $1 / 600$ of the span, when the deflection measurement method in this paper is applied to the actual project, the error should be much smaller than the error of the calculation example in this paper.

This paper only investigates the case of single-span-simple-support bridge, for other types of bridges, more work need to be done.

\section{Acknowledgements}

This research is supported by the characteristic innovation project for colleges in Guangdong province (2019GKTSCX052).

\section{Conflicts of Interest}

The authors declare no conflicts of interest regarding the publication of this paper.

\section{References}

[1] He, C. (2016) Design and Implementation of Real-Time Monitoring System for Bridge Alignment Based on Inclination Sensor. Diss. Ph.D. Thesis, Nanjing University of Posts and Telecommunications, Nanjing.

[2] Ge, L.Q. (2016) Bridge Structure Health Monitoring and Condition Assessment Based on Corners. Ph.D. Thesis, Southeast University, Nanjing.

[3] Li, H.W., Zeng, G.L., Hou, L.Q., Leng, Z.P. and Jiang, X.L. (2010) Research on Practical Methods for Measuring Bridge Deflection Using Inclination Sensors. Journal of Disaster Prevention and Mitigation Engineering, 275-278.

[4] Yang, X.S., Hou, X.M., Liao, Z.P. and Huang, Z.P. (2002) A New Method of Bridge Deflection Measurement. China Civil Engineering Journal, 92-96.

[5] Yin, J., Zhang, Y.F. and Yang, C. (2017) Research on Application of Inclinometer in Bridge Health Monitoring System. Modern Transportation Technology, 14, 42-44.

[6] Yao, J.C., Yang, Y.Q., Wang, W., Liu, P.H., Dong, Z.S., Yin, J., et al. (2018) A Bridge Deflection Test Method and System Based on Inclinometer. https://xueshu.baidu.com/usercenter/paper/show?paperid=1n0m0mf0a14b0690jq64 $\underline{0 \text { e202h280074\&site }=\text { xueshu_se }}$

[7] CCCC Highway Planning and Design Institute (2012) Standard of the Ministry of Communications of P. R.C. Specifications for Design of Highway Reinforced Concrete and Prestressed Concrete Bridges and Culverts (JTG 3362-2018). China Com- 
munications Press, 2018.

[8] Zhang, D.Y., Jin, X., Ding, H.P. and Kong, G. (2005) Application of Ansys/ls-Dyna in Earthquake Engineering. Earthquake Engineering and Engineering Vibration, 25, 170-173. 